\title{
Nonlinear Phenomena and Deterministic Chaos in Systems With Vanadium Dioxide
}

\author{
A.A. Velichko, A.L. Pergament, G.B. Stefanovitch and P.P. Boriskov
}

\begin{abstract}
The switching effect with current-controlled negative resistance ( $S$-shaped voltage-current characteristics) associated with the metal-insulator transition in vanadium dioxide is studied. The experiment shows that the switching devices exhibit complex nonlinear behaviors, including $1 / f$-type electronic noise, the effect of burst noise, stochastic resonance, period-adding sequences with intermittent chaos. In addition, deterministic chaos in the region of negative differential resistance is observed and, near the threshold point, the intrinsic dimension of the attractor is $d=2.3$. Mechanisms of these phenomena are discussed. The analysis allows concluding that the noise and chaotic effects should obviously be taken into account in applications of $\mathrm{VO}_{2}$ in electronic devices, particularly in thermal sensors and bolometeric detectors.
\end{abstract}

Index Terms - vanadium dioxide, switching, stochastic resonance, deterministic chaos.

\section{INTRODUCTION}

$\mathrm{M}$ ANY semiconductor systems can exhibit complex nonlinear behaviors when they are driven far from thermodynamic equilibrium by applying electric fields. The origin of these electrical instabilities is usually closely linked to a negative differential resistance (NDR). In vanadium dioxide the switching effect with current-controlled NDR is caused by the electric-field-induced metal-insulator transition (MIT), which is stimulated by either thermal or electronic effects [1]. In equilibrium conditions, vanadium dioxide undergoes a metal-insulator phase transition at the transition temperature $T_{\mathrm{t}}=340 \mathrm{~K}$ [2], and above $T_{\mathrm{t}}$ the conductivity abruptly increases by $4-5$ orders of magnitude. Thus, electrical switching due to MIT in $\mathrm{VO}_{2}$-based structures is characterized by the S-shaped voltage-current characteristic: at $V=V_{\text {th }}$ (the threshold voltage) the structure undergoes a transition from a high-resistance (OFF) insulating state to a low-resistance $(\mathrm{ON})$ metallic state.

The metal-insulator transition, like any other phase transition,

Manuscript received May 2, 2014; accepted June 2, 2014. Date of online publication: June 30, 2014

This work was supported by the Strategic Development Program of Petrozavodsk State University $(2012$ - 2016) and the RF Ministry of Education and Science as a part of state program in the scientific field, projects no. № 2014/154, № 3.757.2014/K.

A. A. Velichko (velichko@psu.karelia.ru), P. P. Boriskov (boriskov@psu.karelia.ru) are with the Chair of Electronics and Electroenergetics, A. L. Pergament (aperg@psu.karelia.ru) and G. B. Steafanovitch (gstef@psu.karelia.ru) are with the Chair of Information Measuring Systems and Physical Electronics. Department of Physical Engineering at Petrozavodsk State University, 185910 Petrozavodsk, Russia. is a nonlinear phenomenon in nature. Also, in the case of electrical switching, additional nonlinear effects due to the NDR and current filament formation should take place [3]. Therefore, the $\mathrm{VO}_{2}$ based switching devices are expected to demonstrate complex behaviors, from ordinary noise to deterministic chaos. For example, low-frequency noise at the thermally induced MIT has been studied in [4]-[6], and the noise effects at electrical switching in $\mathrm{VO}_{2}$-based structures in [7], [8]. Chaotic oscillations have been obviously first observed in [7]. The experimental works have been carried out on single crystals [5,8], thin films [6],[9],[10] and ceramics [7].

Chaotic dynamics and noise effects in vanadium dioxide are of considerable interest because of potential technical applications of the material. In addition, these effects receive increased attention due to general fundamental interest both in the nonlinear phenomena themselves, and, in particular, in the problem of metal-insulator transitions, because the noise measurements can give valuable information about the transition mechanism [4-6], [8], [10]. As for the applied aspect, vanadium oxide films are used for fabrication of a variety of electrical and optical devices, including electrical switching elements, temperature sensors, optical storage devices, variable reflectivity mirrors, IR bolometric detectors [11]. The latter field of application is of especial interest [6], [11-13], because vanadium dioxide allows the designing of high-sensitive, spectrally broadband and uncooled bolometers. The study of noise and related phenomena in such devices is obviously an important problem from the viewpoint of the improvement of the bolometric sensor sensitivity and accuracy. In this article we present results of comprehensive experimental study of the noise and chaotic phenomena in $\mathrm{VO}_{2}$ based switching devices.

\section{SAMPLES AND EXPERIMENTAL TECHNIQUES}

In our experiments we used both sandwich MOM (metaloxide-metal) and planar $\mathrm{VO}_{2}$ switching devices. The sandwich devices were fabricated by the anodic oxidation of vanadium metal (vacuum-deposited vanadium layers and foils) [14]. The thickness of oxide films was $\sim 100 \mathrm{~nm}$. Metal aluminum electrodes of $1 \mathrm{~mm}$ in diameter were evaporated onto the surfaces of oxide films to complete the MOM structures. Channels $(\sim 1 \mu \mathrm{m}$ in diameter) consisting of the vanadium dioxide phase were formed in the initial anodic films during electroforming [14]. After the process of electroforming, voltage-current characteristics of the devices became S-shaped (Fig.1). As the temperature increased, the switching threshold voltage $V_{\text {th }}$ decreased to zero at a certain critical temperature. 
This temperature was found to be practically equal to the transition temperature for vanadium dioxide $T_{\mathrm{t}}=340 \mathrm{~K}$ indicating that the switching mechanism was associated with the MIT.

For the planar switching devices, $\sim 100 \mathrm{~nm}$ thick $\mathrm{VO}_{2}$ films on insulating substrates were fabricated by reactive magnetron sputtering. Aluminum electrical contacts for the four-probe measurements were obtained by thermal vacuum evaporation. The structures had $5 \mathrm{~mm}$ gap between Al-contacts. The $V-I$ characteristics of the planar devices were qualitatively the same as those of the sandwich devices, and the threshold voltage in this case was $\sim 100 \mathrm{~V}$.

The experimental setup included a DC power source and different AC generators, an oscilloscope, and a load resistor $R_{\mathrm{L}}$. The latter was connected in series with the sample, and the current was monitored by determining the voltage drop across an additional series resistor $R<<R_{\mathrm{ON}}$, where $R_{\mathrm{ON}}$ is the $\mathrm{ON}$ state resistance. The output signals of $V$ and $I$, via an amplifier and analog-digital converter, were recorded and processed with a PC.

\section{RESUlTS AND DisCUSSION}

\section{A. Intrinsic noise under DC bias and relaxation oscillations}

Under DC bias the devices are characterized by an intrinsic noise both in OFF and in ON states. On the whole, the power spectra vary with frequency as $1 / f^{\alpha}(\alpha=1.1 \pm 0.1)$. Such behavior has been established earlier for vanadium oxide switching structures [8]. It was shown that in the OFF state there was a mixture of a flicker noise and pre-breakdown generation-recombination fluctuations. The low-frequency noise of $1 / f$ type has been also found in thin vanadium dioxide films [6] and in other materials exhibiting MITs ( $\mathrm{La}_{2}$ $\left.{ }_{x} \mathrm{Sr}_{x} \mathrm{MnO}_{3}, \mathrm{Fe}_{3} \mathrm{O}_{4}[15]\right)$ as well.

In the ON state, an additional noise, which is probably associated with the fluctuations in the current filament, contributes to the overall spectrum. Near the thresholds and in the NDR region, the oscillations become more complex. In this case, the output signal contains a component corresponding to deterministic chaos. The results of the study of deterministic chaos will be discussed in more details below, in Section 3.E.

If the value of the DC voltage $V_{0}$ is sufficiently high, relaxation oscillations with the amplitude $V_{\mathrm{r}}=V_{\mathrm{th}}-V_{\mathrm{h}}$ appear if the load line intersects with the I-V curve in a unique point (point $B$ in Fig.1) in the NDR region. The period of this oscillation is proportional to the relaxation time $\tau=a R C$ (where $\mathrm{C}$ is the capacitance of the device, and $a$ is the parameter depending on the $R_{\mathrm{OFF}} / R_{\mathrm{L}}$ ratio) and it depends logarithmically on $V_{0}$ :

$T_{\mathrm{r}} \approx \tau \ln \left[\left(V_{\mathrm{o}}-a V_{\mathrm{h}}\right) /\left(V_{\mathrm{o}}-a V_{\mathrm{th}}\right)\right]$.

The equation (1) can be easily deduced form the time dependence of $V$ during the capacity charging. The dependences of the mean value and dispersion of $T_{\mathrm{r}}$ on $V_{0}$ are

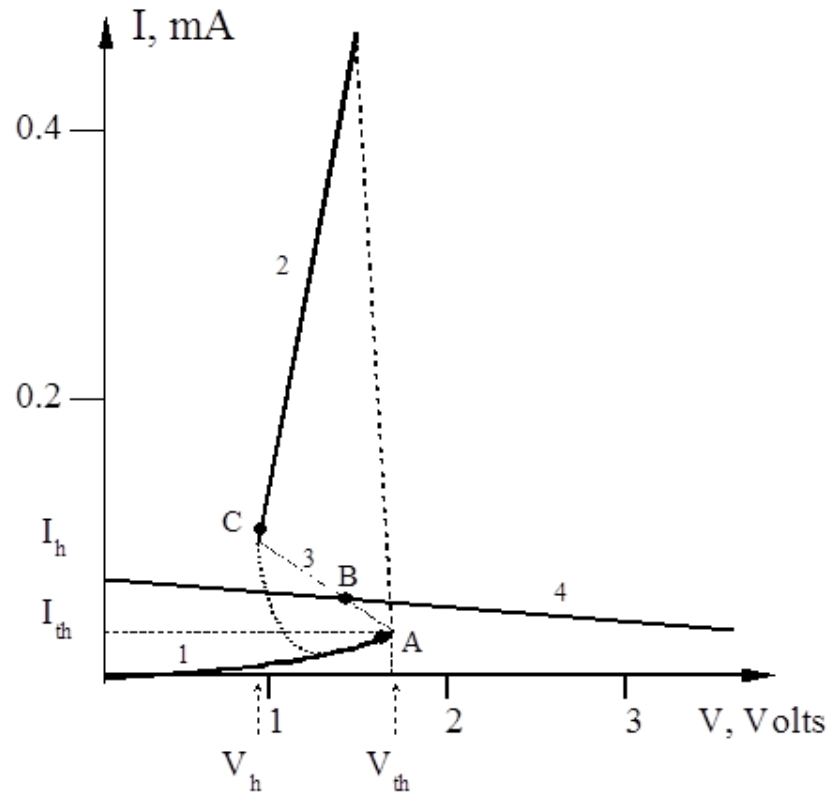

Fig. 1. I-V characteristic of the $\mathrm{VO}_{2}$ sandwich structure and the main definitions (1- OFF state, 2- ON state, 3- NDR region, 4- load line; $V_{\text {th }}$ and $I_{\text {th }}$ - threshold voltage and current, $V_{\mathrm{h}}$ and $I_{\mathrm{h}}$ - holding voltage and current).

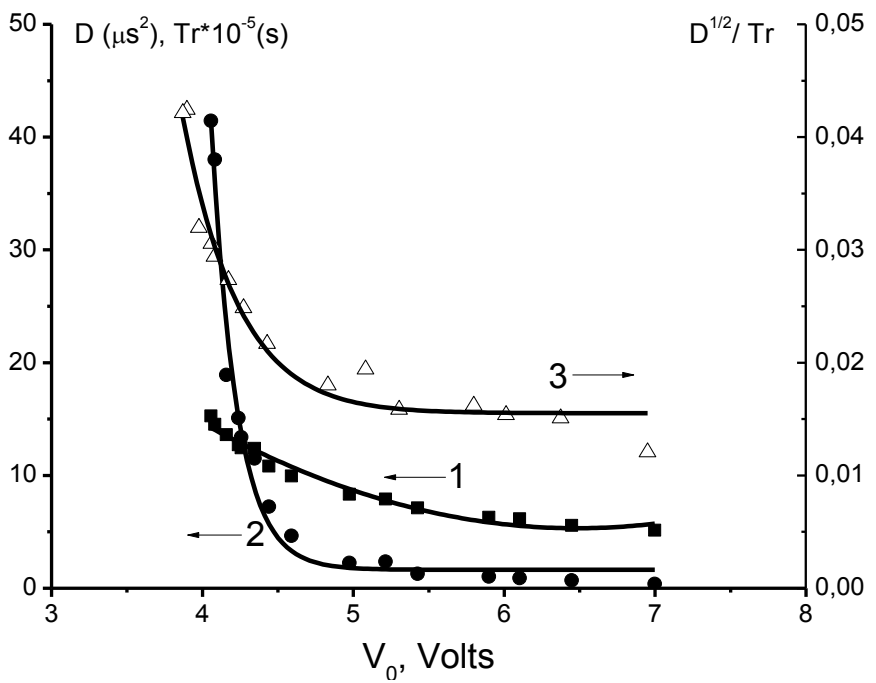

Fig. 2. Relaxation oscillations period $T_{\mathrm{r}}(1)$, its dispersion $D(2)$ and relative error $D^{1 / 2} / T_{r}(3)$ as functions of applied voltage $V_{0}\left(V_{\mathrm{th}} \approx 4 \mathrm{~V}\right)$.

shown in Fig.2. It should be noted that the minimum error can be made much less than 1\% (i.e. less than that shown in Fig.2, curve 3 ) by carefully adjusting the circuit parameters: $R_{\mathrm{L}}, V_{0}$, etc.

Since an external noise always presents along with the internal one, we further investigate how it affects the $\mathrm{VO}_{2}$ switching devices. 

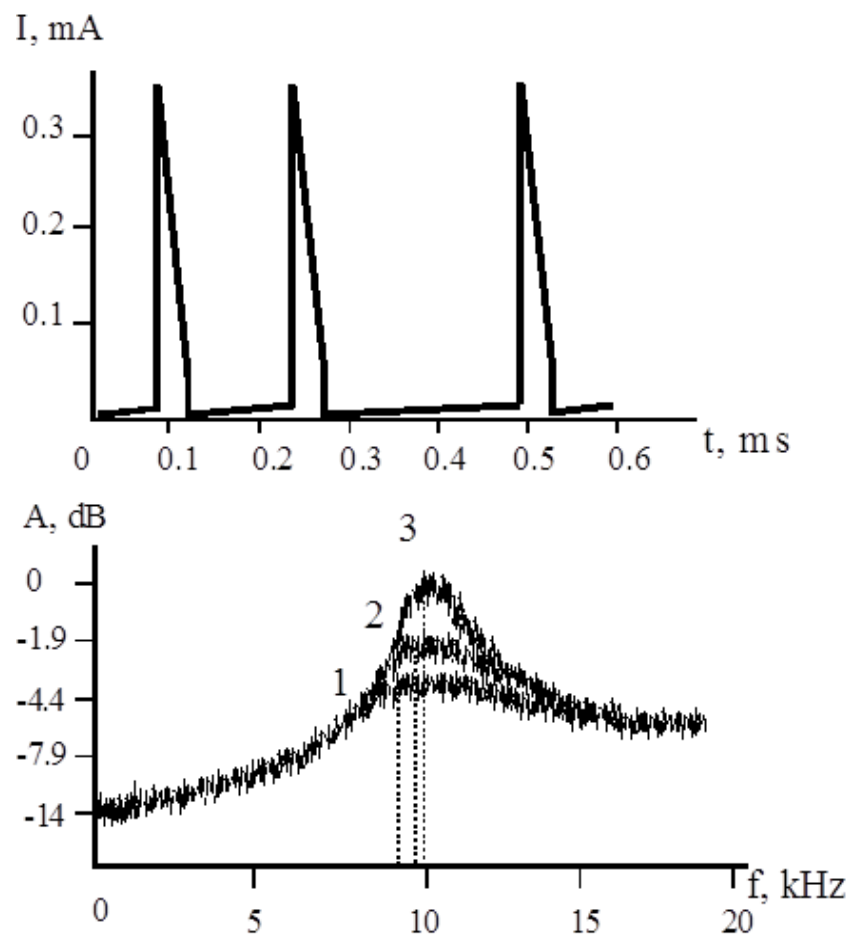

Fig. 3. Chaotic current oscillations induced by a slight external noise (a) and their spectrum (b) at different DC voltages $\left(V_{11}<V_{12}<V_{13}\right)$.

\section{B. Modeling of the switch response to external noise}

If the structure is stabilized near the threshold point $\left(V=V_{1}<V_{\text {th }}\right.$ and $\left[V_{\text {th }}-V\right] / V \rightarrow 0:$ Fig. 1 , point $\left.A\right)$, a small voltage fluctuation can lead to formation of an intense current pulse. This phenomenon is similar to the burst noise [16], where small noise due to the random change of internal or external parameters is transformed to the noise of giant magnitude.

For modeling of the device response to external perturbations, we selected the operating point of the circuit in the under-threshold regime $\left(V=V_{1}\right)$, and affected the system by the external noise from a Gaussian noise generator. This generator had the frequency band up to $20 \mathrm{kHz}$ and amplitude $V_{\mathrm{n}}=0.01-0.2 \mathrm{~V}$ and was connected in series with the DC power supply. Since in this case the scheme has a sharp response, the external noise results in random switching with large current and voltage pulse amplitudes. The output current signal represents a sequence of spikes with the chaotically varied period (Fig.3). The spectrum of the output signal has a maximum in the vicinity of the relaxation frequency $f_{\mathrm{r}}=1 / T_{\mathrm{r}}$. The maximum rises with increasing $V_{1}$, i.e. the oscillations with $f=f_{\mathrm{r}}$ are the most probable. Also, a slight shift of the spectrum toward the high-frequency region is observed, that is associated with the dependence of the relaxation frequency on $V_{0}$ (see Eq.(1)).

\section{Stochastic resonance}

Further, into the circuit operating in a mode of random oscillations, we have added a weak periodic signal with the frequency $\mathrm{f}_{0}$ from a sin-wave generator connected in parallel with the DC source and with the noise generator. In this case a maximum at $f=f_{0}$ is observed in the spectrum. The height of this maximum depends on the noise intensity $V_{\mathrm{n}}$ at a constant voltage $V_{1}$, and also it depends on $V_{1}$ at a constant noise level. Thus, at certain parameters of the circuit, the amplitude amplification of a harmonic signal and an increase in a signalto-noise ratio (SNR) are observed. We interpret this observation as the effect of stochastic resonance.

The phenomenon of stochastic resonance has attracted much attention over the last decade being a distinguished example of nontrivial noise-induced effect in nonlinear systems and a mechanism of noise-controlled selforganization in complex systems. In general, stochastic resonance is the result of the change of the metastable state if a relatively small and slowly changing deterministic perturbation is added to the system [17]. If this perturbation is periodic, then under certain assumption, the system will perform a motion, which is close to a large amplitude oscillation with the same period or with a period proportional to the period of deterministic perturbation.

The dependence of SNR on $V_{1}$ can be treated as a change of the switching dynamics depending on the value of a potential threshold of a bistable switching system. Note that both the gain factor and the maximum of SNR increase as the frequency $f_{0}$ tends to the relaxation frequency of the switch.

Similar behavior is observed if the source of external noise is turned off. We conclude therefore that in the absence of an external noise, the internal switch noise serves as the disorder factor in experiments on stochastic resonance, and the optimum regime of amplification is reached by variation of the voltage $V_{1}$.

\section{Generation of stochastic oscillations}

For a sufficiently large amplitude of the periodic signal, a new nonlinear effect in stochastic resonance has been found. In these experiments, a sin-wave generator was connected in series with the DC source. In the presence of a weak internal noise it is possible to observe the phenomenon of locking the mean switching frequency [18] - see Fig.4. At first the frequency of switching coincides with the signal frequency $\Omega$. Then, as the $\Omega$ increases over the natural system frequency $f_{\mathrm{r}}$, the switching period becomes twice as greater in comparison with $T_{\mathrm{L}}=1 / \Omega$ (Fig.4,c). Between these two states, a chaotic regime appears with the phase diagram of $I$ and $V$ shown in Fig.4,b. Thus, there exist two limit cycles, between which the phase trajectory moves chaotically and fills in the space between them. As the frequency continues to increase, the described process is repeated, i.e. the oscillations with $T=N T_{\mathrm{L}}$ $(N=3,4,5 \ldots)$ appear. The same transition between different oscillating behaviors is observed when varying the amplitude of the sin-wave signal or the DC voltage $V_{0}$, not only the frequency $\Omega$.

The above-described chaotic behavior represents the intermittent chaos [19], i.e. the random transitions between two limit cycles (attractors) during the period-adding process. 

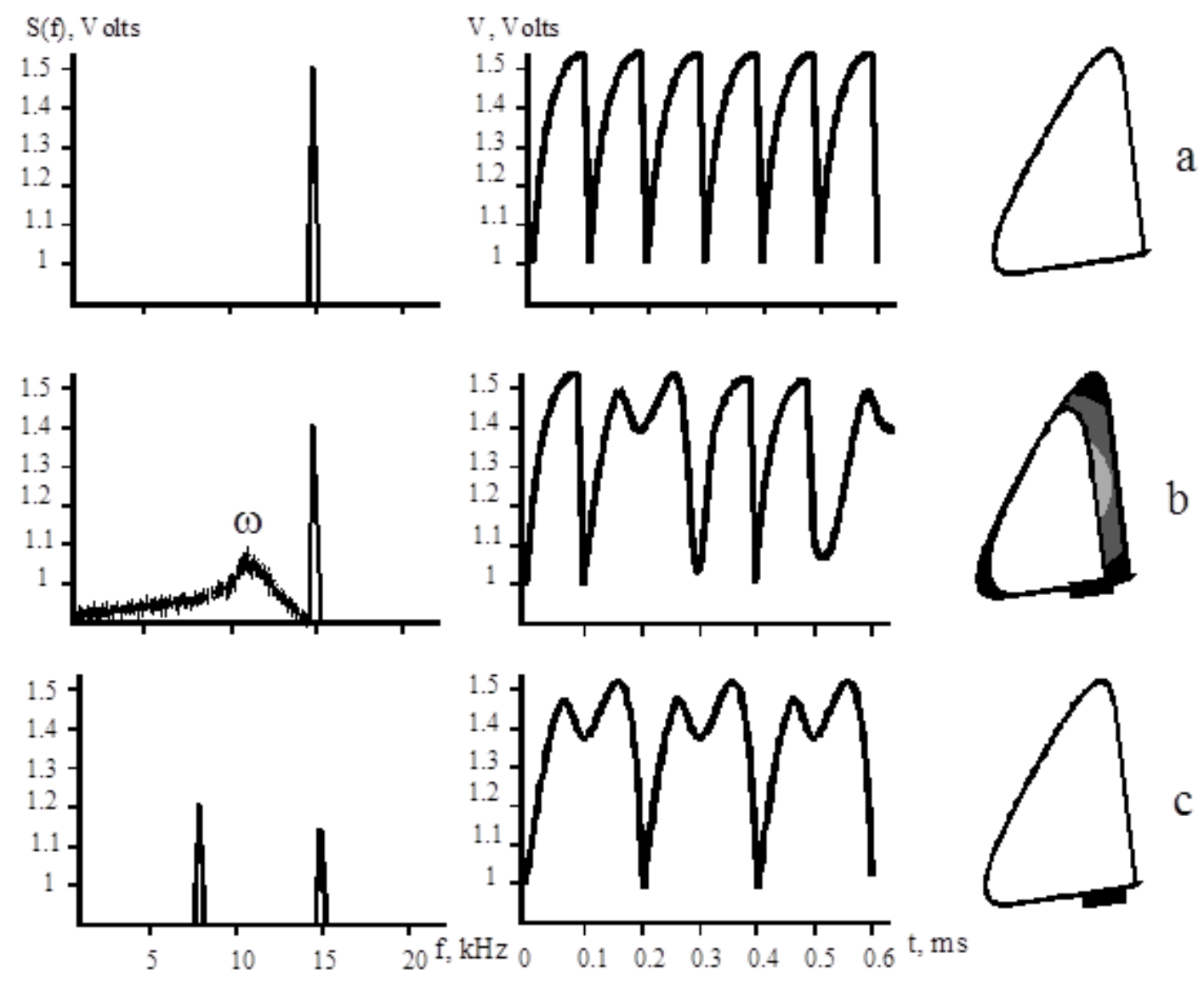

Fig. 4. Oscillation spectra, voltage oscillograms, and phase portraits (I-V orbits) for different values of $\Omega$ : (a) $\Omega_{1}$, frequency locking; (b) $\Omega_{2}$, chaotic oscillations; and (c) $\Omega_{3}$, period doubling. $\Omega_{1}<\Omega_{2}<\Omega_{3}$.

One can assume that the nature of chaotic oscillations is conditioned by the presence of an internal noise in the dynamically unsteady bistable system. In this case the amplification of this internal noise occurs. The spectrum of arising stochastic oscillations represents the dependence of the gain on frequency. The maximum of this spectrum lies near a certain frequency $\omega$ (Fig.4, b) which depends on the parameters of the oscillating circuit.

When adding a gentle periodic signal with frequency $\mathrm{f}_{0}$ from a sin-wave generator (like in the above-described experiments, Section 3.C), the picture similar to the phenomenon of stochastic resonance is observed. An internal noise of the structure plays the role of a fluctuation signal. The weak periodic component is enhanced in amplitude. Also, the SNR increases because of the predominant initiation of oscillations synchronously with the signal. The greatest gain $(\sim 100)$ is reached in the region of $f \approx \omega$. The frequency $\omega$ thus can be interpreted as the most probable frequency of random switching events.

\section{E. Deterministic chaos}

Deterministic chaos, the phenomenon in which a certain system shows unpredictable behavior although it is rigorously described by nonlinear differential equations, has recently attached interests in many areas of science and engineering [19]. In the field of electronics, circuits containing the NDR devices (such as, e.g., thyristors [20]) are known to exhibit chaotic behaviors.

In this section we present the results of investigation of chaotic behavior of $\mathrm{VO}_{2}$ based switching devices in planar configuration. The latter allow us to use the four-probe measurement technique and thereby exclude the systematic error connected with the contact resistance.

In these experiments the device was DC biased and the operating point was fixed within different regions of the $I-V$ characteristic (points $A, B, C$ in Fig.1). A chaotic AC component of the output signal was recorded by a PC, and the data were treated by numerical methods (spectrum calculations, filtration, Fourier transformation, and calculations of the correlation dimension using the Grassberger-Proccacia algorithm [21]).

The correlation dimension $v$ of the attractor was determined [19], [21] by using the scaling dependence $C(r) \sim r^{v}$ at $r \rightarrow 0$ where the correlation integral with respect to arbitrary distance $r$ is given by

$$
\begin{aligned}
& C(r)=\lim \frac{1}{N^{2}} \sum_{i=1}^{N} \sum_{j=1}^{N} H\left(r-\left|x_{i}-x_{j}\right|\right), \\
& N \rightarrow \infty(i \neq j)
\end{aligned}
$$

where $H$ is the Heaviside function, $N$ is the number of 
points and $|\boldsymbol{X} i-\boldsymbol{X} j|$ is the distance between two points in the attractor. Fig.5 shows the procedure by which the value of $v$ was obtained for different embedding dimensions $P$. The obtained results can be summarized as follows:

1. There is no deterministic chaos in the OFF state; the fractal dimension is relatively large, $d>5$ (Fig.5,a) indicating the stochastic nature of noise.

2. For the oscillations in the NDR region near the threshold point, the intrinsic dimension of the attractor was obtained $d=2.3$ (for $P=5$ ), as shown in Fig.5,b. Such a fractal number of dimension is an attribute of deterministic chaos [19]. The value $d=2.3$ means that the oscillations are in 3D phase space and they are described by at least three differential equations.

3. For higher currents, when the operating point is still in NDR, but it is relatively far from the threshold, the fractal dimension is $2<d<3$ (Fig.5,c). This indicates that oscillations appear to be a mixture of deterministic chaos and stochastic noise.

It is critical to note that in the case of deterministic chaos, unlike uncorrelated noise, an efficient signal extraction from a signal-noise mixture is possible (at least in principle). In recent years, several techniques have been developed for forecasting behavior of dynamic systems and for separation of deterministic and random noise. These techniques are based on algorithms of time-series forecasting [22-25]. The underlying principle of such time-series forecasting and separation is to predict future values of a time series by consulting a catalog of how the system evolved at other times when initial conditions are either similar or slightly different due to noise. The same techniques might be obviously utilized for separation of the deterministic noise and informative signal. Therefore, on the basis of computing simulation, using, e.g., the algorithm of short-time forecasting of chaotic time series [22], [23], it is possible to diminish the SNR and thereby to improve the accuracy of $\mathrm{VO}_{2}$ electronic devices and sensor systems.

\section{CONCLUSION}

The experiment shows that the switching devices exhibit complex nonlinear behaviors, such as: the burst noise, stochastic resonance, period-adding sequences with intermittent chaos, and deterministic chaos. It is quite evident that both the MIT, and the NDR are responsible for these effects, as well as for the $1 / f$-type noise. Also, current filament formation contributes to the process. Therefore, the study of these phenomena is necessary for further understanding the physical mechanisms of MIT and switching in $\mathrm{VO}_{2}$.

The effects described should be obviously taken into account when working out the electronic devices (as thermal sensors and bolometers) based on vanadium dioxide. It would be rather tempting to utilize the sharp temperature dependence of resistivity at $T=T_{\mathrm{t}}$ (or, for the switching devices, the $\mathrm{I}(\mathrm{V})$ dependence within the NDR region), which could ensure enormous sensitivity.
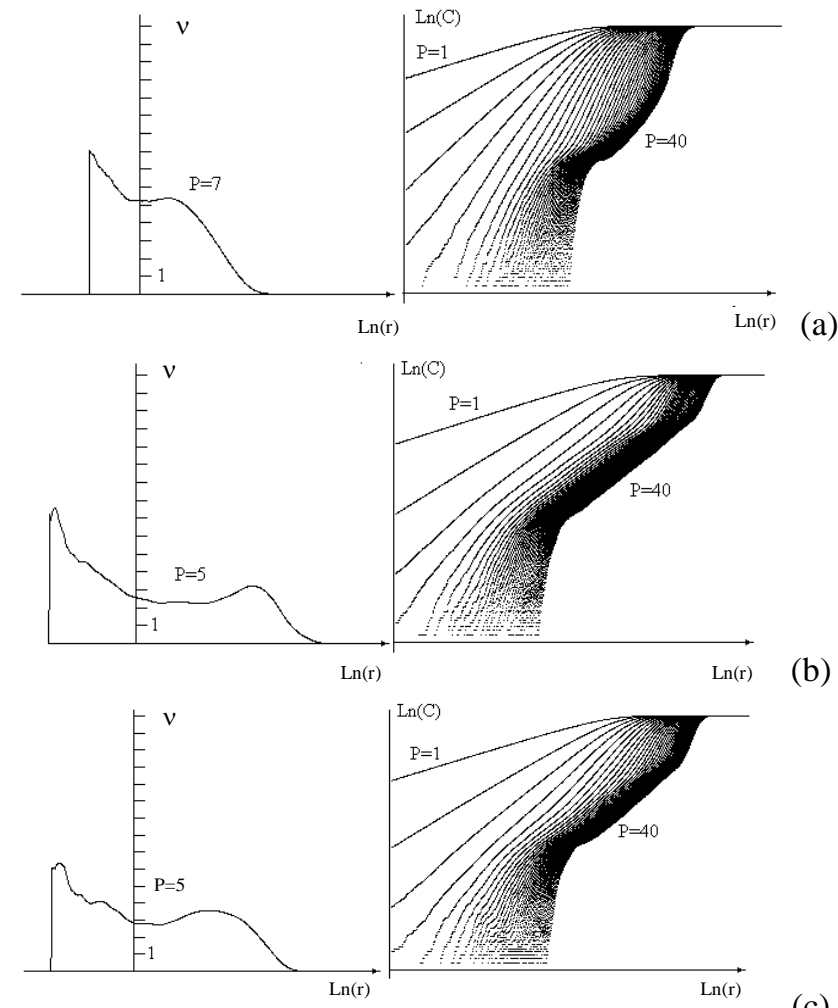

(c)

Fig. 5. The scaling factor $v$ (left) and the logarithmic scaling $\operatorname{Ln}(C)$ (right) from $\operatorname{Ln}(r)$ for different embedding dimensions $P$ : (a) $P=7, I=0.2 \mathrm{~mA}$; (b) $P=5, I=0.2 \mathrm{~mA}$; (c) $P=5, I=1 \mathrm{~mA}$.

(scale) for

Unfortunately, this method turns out to be not efficient because of the hysteresis effects [11] and also because of chaotic behavior for low thermal cycling rates [6]. The latter obstacle might nevertheless be avoided by, e.g., the singlepulse excitation [6], [12]. However, as shown in Section 3.B of the present paper, the effect of burst noise will affect negatively the device accuracy in this case. A different promising approach is based on the $f_{\mathrm{r}}(T)$ dependence [14]. The temperature sensor (or bolometric detector) with the frequency output, proposed in Ref.[14], seems to be more appropriate for technical applications, because the noise-induced error might be made sufficiently small in this case (see Fig.2).

Also, as was discussed in Section 3.E above, in the case of deterministic chaos, it is possible to extract the informative signal by the methods of forecasting of chaotic time series [22], [23].

Finally, it is shown that vanadium dioxide appears to be an ideal model object for demonstration and investigation of diverse nonlinear phenomena, which are observed simultaneously and, moreover, can interplay among themselves.

\section{REFERENCES}

[1] A. L. Pergament, G. B. Stefanovich, A. A. Velichko, "Oxide Electronics and Vanadium Dioxide Perspective: A Review", Journal on Selected Topics in Nano Electronics and Computing, vol. 1, pp. 24-43, 2013.

[2] N. F. Mott, Metal-Insulator Transitions, 2nd ed., Taylor \& Francis, London, 1990. 
[3] E. Schöll, Nonequilibrium Phase Transitions in Semiconductors, Springer-Verlag, Berlin, 1987.

[4] A. A. Kokin, G. B. Mikhailov, On low frequency current fluctuations in systems with semiconductor-metal phase transition, Phys. Solid State, vol. 18, pp. 1970-1974, 1976.

[5] V.N. Andreyev, B.P. Zakharchenya, Yu.S. Kapshin, V.A. Noskin, F.A. Chudnovsky, Low-frequency noise in vanadium dioxide experiencing a metal-dielectric phase transition, Soviet Physics JETP, vol. 52, no. 4, pp. 684-688, 1980.

[6] L. A. L. de Almeida, G. S. Deep, A. M. N. Lima, H. Neff, Thermal dynamics of $\mathrm{VO} 2$ films within the metal-insulator transition, Appl. Phys. Lett. vol. 77, pp. 4365 - 4367, 2000.

[7] Y. Taketa, R. Furugochi, Switching and oscillation phenomena in SnO2-VOX-PdO ceramics, Appl. Phys. Lett. vol. 31, pp. 405-406, 1977.

[8] A. Alekseyunas, V. Bareikis, V. Bondarenko, Yu. Liberis, Current fluctuations and switching time in vanadium dioxide single crystals, Fizika Tverdogo Tela, vol. 20, no 7, pp. 1980-1984; For English translation see the journal Sov. Phys. - Solid State.

[9] V.S. Aliev, S.G. Bortnikov, M.A. Dem'yanenko, Signal amplification under stochastic resonance in vanadium dioxide film, Tech. Physics Letters, vol. 38, no 11, pp 965-968, 2012.

[10] A.A. Velichko, G.B. Stefanovich, A.L. Pergament, P.P. Boriskov, Deterministic noise in vanadium dioxide based structures, Tech. Physics Letters, vol. 29, no. 5, pp. 435-437, 2003.

[11] H. Jerominek, F. Picard, D. Vincent, Vanadium oxide films for optical switching and detection, Optical Engineering, vol. 32, pp. 20922099, 1993.

[12] V. Y. Zerov, Yu. V. Kulukov, V. N. Leonov, V. G. Malyarov, I. A. Khrebtov, I. I. Shaganov, Features of operation of the bolometer on the basis of vanadium dioxide film in temperature range including phase transition, J. Opt. Technol. vol. 66, pp. 387-391, 1999.

[13] K. Prokhorov, A. Velichko, A. Pergament // Sensor element for a thermal imaging matrix based on vanadium oxide film // The 25th Nordic Semiconductor Meeting. pp. 54-56 (2013). http://physics.aalto.fi/ filip/abstracts/35 Prokhorov POSTER.pdf.

[14] A. L.Pergament, G. B.Stefanovich, and F. A.Chudnovskii, Metalsemiconductor phase transition and switching effect in oxides of transition metals, Phys. Solid State, vol. 36, no. 10, pp. 1590-1597, 1994.
[15] B. Raquet, J. M. D. Coey, S. Wirth, S. Von Molnar, 1/f Noise in the half-metallic oxides $\mathrm{CrO} 2, \mathrm{Fe} 3 \mathrm{O} 4$ and $\mathrm{La} 2 / 3 \mathrm{Sr} 1 / 3 \mathrm{MnO} 4$. Phys. Rev. B, vol. 59, pp. 12435-12440, 1999.

[16] M.J. Buckingham, Noise in Electronic Devices and Systems, John Wiley \& Sons, New York, 1983.

[17] M. I. Freidlin, Quasi-deterministic approximation, metastability and stochastic resonance, Physica D, vol. 137, pp. 333-352, 2000.

[18] B. Shulgin, A. Neiman, V. Anishchenko, Mean switching frequency locking in stochastic bistable systems driven by a periodic force, Phys. Rev. Lett. vol. 75, pp. 4157-4160, 1995.

[19] F. C. Moon, Chaotic Vibrations, John Wiley \& Sons, New York, 1987.

[20] K. Hoh, Y. Yasuda, Electronic chaos in silicon thyristor, Jpn. J. Appl. Phys. vol. 33, pp. 594 - 598, 1994.

[21] P. Grassberger, I. Proccacia, Characterization of strange attractors, Phys. Rev. Lett. vol. 50, pp.346-349, 1983.

[22] J.D. Farmer, J.J. Sidorowich, Predicting chaotic time-series, Phys. Rev. Lett. vol. 59, pp. 845-848, 1987.

[23] J.D. Farmer, J.J. Sidorowich, Exploiting chaos to predict the future and reduce noise, Y.D. Lee. World Scientific, Singapore, 1988.

[24] M. Casadagli, Nonlinear prediction of chaotic time series, Physica D, vol. 35, pp. 335-356, 1989.

[25] T. Sauer, Time Series Prediction Using Delay Coordinate Embedding, Time series Prediction: Forecasting the Future and Understanding the Past. Eds. A.S.Weigend, N.A.Gershenfeld, Addison-Wesley, 1993. 